Comment. Math. Helv. 74 (1999) 376-397

(C) 1999 Birkhäuser Verlag, Basel

0010-2571/99/030376- $22 \$ 1.50+0.20 / 0$

Commentarii Mathematici Helvetici

\title{
Alternating knots satisfy strong property $\mathbf{P}$
}

\author{
Charles Delman and Rachel Roberts ${ }^{1}$
}

\begin{abstract}
Suppose a manifold is produced by finite Dehn surgery on a non-torus alternating knot for which Seifert's algorithm produces a checkerboard surface. By demonstrating that it contains an essential lamination, we prove that such a manifold has $\mathbb{R}^{3}$ as universal cover and, consequently, is irreducible and has infinite fundamental group. Together with previous work of Roberts, who proved this result in the case of alternating knots for which Seifert's algorithm does not produce a checkerboard surface, and Moser, who classified the manifolds produced by surgery on torus knots, this paper completes the proof that alternating knots satisfy Strong Property P.
\end{abstract}

Mathematics Subject Classification (1991). Primary 57M25; Secondary 57R30.

Keywords. Alternating knot, essential lamination, Dehn surgery, property P, 3-manifold.

\section{Introduction}

In this paper we complete the proof that alternating knots satisfy Strong Property P. A knot $k$ is said to satisfy Property $P$ if no finite surgery along $k$ yields a simplyconnected 3-manifold. In [G7], Gabai introduced the following stronger notion (which clearly implies Property P):

Definition 1.1. A knot $k$ is said to satisfy Strong Property $P$ if no finite surgery along $k$ yields a 3 -manifold with a simply-connected summand.

Objects of co-dimension one have long been used to obtain information about the fundamental groups of 3-manifolds. The notion of an essential lamination was developed to bridge the gap between incompressible surfaces, at one extreme, and taut foliations, at the other. Briefly, a lamination is a closed subset which is foliated by leaves of dimension two; it is essential if these leaves are suitably embedded (as precisely described by Definition 3.1). Gabai and Oertel proved the following theorem, which shows that existence of an essential lamination implies Strong Property P.

\footnotetext{
${ }^{1}$ Research supported in part by an NSF Postdoctoral Fellowship.
} 
Theorem 1.1. [GO] Let $M$ be a closed oriented 3-manifold with an essential lamination. Then $M$ is irreducible and has infinite fundamental group and universal cover $\mathbb{R}^{3}$.

For convenience in discussing the alterating knots, we make the following definition.

Definition 1.2. We shall call an alternating knot $k$ checkerboard if it possesses a regular alternating projection $\pi$ such that one of the checkerboard surfaces described by $\pi$ is an essential (that is, both incompressible and $\partial$-incompressible) Seifert surface for $k$. Otherwise, we shall call $k$ noncheckerboard.

The Tait flyping theorem [MT] reveals that $k$ is checkerboard if and only if every reduced alternating projection for $k$ yields an essential checkerboard Seifert surface. Note that this is exactly the surface given by Seifert's algorithm. We also observe that the only alternating torus knots are the $(2, n)$ torus knots, all of which are checkerboard knots.

In earlier work, Roberts proved:

Theorem 1.2. $[\mathrm{R}]$ Let $k$ be a noncheckerboard alternating knot. Then every finite Dehn surgery along $k$ produces a manifold containing a taut foliation.

In this paper, we extend the above result by proving the following

Main Theorem (4.1). Every finite Dehn surgery along a nontorus checkerboard alternating knot produces a manifold containing an essential lamination.

Together with Moser's explicit categorization of the manifolds obtained by surgery on torus knots [Mo], from which it is evident that they satisfy Strong Property P, Theorems 1.1, 1.2 and 4.1 yield the conclusion claimed in the title.

The proof of the Main Theorem proceeds as follows. First, we reduce the problem to the case of prime knots by showing that every composite knot (whether alternating or not) satisfies Strong Property P. We do this by constructing, in the exterior of each composite knot, an essential lamination without boundary which remains essential after every Dehn surgery. Following [D2], we call such a lamination persistent. The method of construction is very elementary and is distinct from the methods used for prime alternating knots.

Next, let $k$ be a prime alternating knot with tubular neighborhood $N(k)$. In this case, we construct the essential laminations of the Main Theorem using one of two methods, each of which begins with an essential spanning surface for $k$. The first method uses this spanning surface to generate a set of essential laminations in $S^{3} \backslash \stackrel{\circ}{N}(k)$ meeting $\partial N(k)$ in simple closed curves. These laminations cap off to give essential laminations in the manifolds obtained by surgery on their boundary 


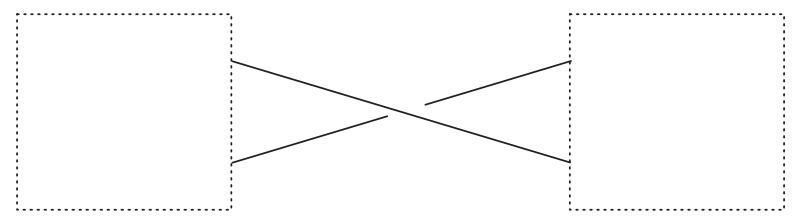

Figure 1.

Nugatory crossing.

curves; for this reason, we extend the term "persistent" to apply to them. For a family of examples, this first approach fails; for each knot in this family, we show the existence of a persistent lamination without boundary, as developed in [D2].

The remainder of the article is organized as follows. In Section 2 we develop the necessary understanding of spanning surfaces in alternating link complements. In Section 3 we recall the necessary facts about laminations, present the proof that the complement of every composite knot contains a persistent lamination, and describe the two methods of constructing laminations using the spanning surfaces developed in Section 2. In Section 4 we show the existence, in the complement of each checkerboard nontorus alternating knot, of either a set of persistent laminations with boundary (covering all surgery curves) or else a persistent lamination without boundary, thus proving the Main Theorem.

\section{Spanning surfaces}

\subsection{Checkerboard surfaces}

A surface will be called essential if it is both incompressible and $\partial$-incompressible. (We additionally assume that its complement is irreducible and that the intersection of the boundary of the ambient manifold with the complement of the surface is incompressible in the complement of the surface; $c f$. Definition 3.1. These additional conditons will be obvious for every surface we consider.)

Let $l$ be a link in $S^{3}$ with regular projection $\pi . \pi$ is called reduced if it contains no nugatory crossing (see Figure 1 ).

Let $S$ and $T$ denote the two checkerboard surfaces associated with $\pi$. In general, $S$ and $T$ are neither essential nor orientable. However, when $l$ is alternating we can say the following.

Lemma 2.1. Let $l$ be a nonsplit link with reduced alternating projection $\pi$. Then both checkerboard surfaces, $S$ and $T$, for $\pi$ are essential.

Proof. We view $l, S$ and $T$ as lying in the projection sphere except near crossings. $S$ and $T$ intersect at each crossing in a vertical arc (with endpoints on $l$ at the preimages of the crossing points and midpoint in the projection sphere); these 


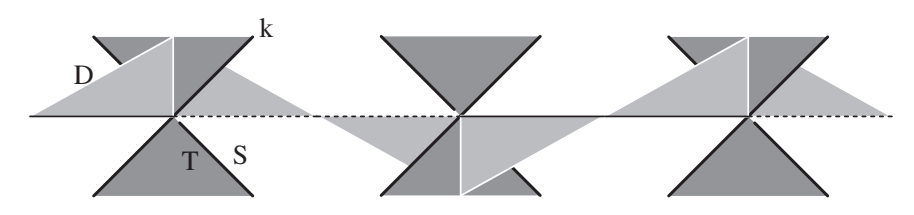

Figure 2.

Arcs of $\mathcal{D} \cap T$ with endpoints on $\partial \mathcal{E}$.

arcs partition $S$ and $T$ into discs, naturally viewed as polygons, whose boundary consists of arcs of $S \cap T$ alternating with arcs on $\partial N(l)$. These polygons, or "tiles", lie in the projection sphere except for small regions containing half of each arc of $S \cap T$. We may assume that no tile has two sides lying on the same arc of $S \cap T$, since the crossing corresponding to this arc would have to be nugatory.

Suppose, without loss of generality, that $S$ is inessential. Since the boundary of the knot complement is a torus, and $S$ is not a $\partial$-incompressible annulus, $S$ must in fact be compressible. By a standard innermost disc argument, we may assume that a compressing disc for $S$ intersects $T$ in a collection of arcs. Among all such compressing discs, let $\mathcal{D}$ be such that the number of $\operatorname{arcs}$ in $\mathcal{D} \cap T$ is minimal.

$\mathcal{D}$ intersects the tiles of $T$ in arcs with endpoints in $S \cap T$. No $\operatorname{arc}$ of $\mathcal{D} \cap T$ has endpoints on the same side of a tile, for, if there were such an arc, consider an innermost one. This arc, together with an arc in $S \cap T$, bounds a disc in $T$. Surgery along this disc produces two discs with boundary in $S$, each intersecting $T$ in a smaller number of arcs, and at least one of these discs must be essential, contradicting our minimality assumption. Similarly, no $\operatorname{arc}$ of $\partial \mathcal{D}$ joins a side of a tile to itself, since an innermost such arc could be removed by isotoping $\mathcal{D}$, reducing the number of $\operatorname{arcs}$ in $\mathcal{D} \cap T$.

From the above description, we see that $\partial \mathcal{D}$ lies in the projection sphere except, possibly, near some crossings. Let $\mathcal{E}$ be an innermost disk bounded by the projection of an arc of $\partial \mathcal{D}$ (or all of $\partial \mathcal{D}$, if its projection has no self-intersections).

We claim that no $\operatorname{arc}$ of $\mathcal{D} \cap T$ lies in $\mathcal{E}$. Note that arcs with endpoints on $\partial E$ must alternately lie outside and inside $\mathcal{E}$ (see Figure 2). Suppose there is an arc lying in $\mathcal{E}$; then some such arc would be innermost on $\mathcal{D}$, and its ends would of necessity violate the alternation rule just stated.

From the above claim, it follows that $\partial \mathcal{E}$ lies in a single tile, which is impossible since it would have to be an arc joining two sides lying at the same crossing. Thus we conclude that no compression exists and $S$ is essential.

Lemma 2.2. Let $\pi$ be a reduced alternating projection of a checkerboard link. Let $T$ be the checkerboard surface for $\pi$ dual to the one given by Seifert's algorithm. Then $T$ is nonorientable.

Proof. If $T$ is orientable, then it induces an orientation on the link it spans. This is impossible since near a crossing we see the behaviour of Figure 3 (up to crossing 


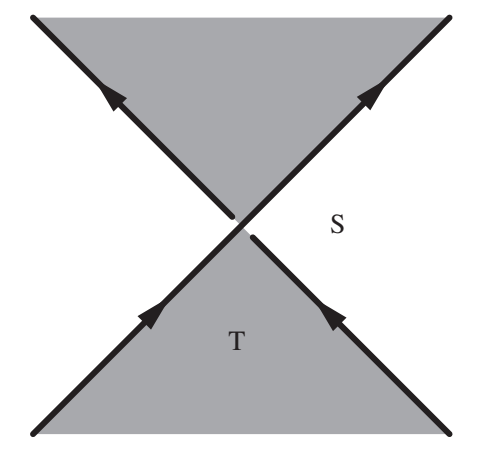

Figure 3.

Orientation of $k$ near crossings.

sign). Hence $T$ is necessarily nonorientable.

As well as checkerboard surfaces arising from alternating link projections, we will consider Murasugi sums of two such surfaces. We recall that a surface $F$ is said to be a Murasugi sum of surfaces $F_{1}$ and $F_{2}$ if there is a 2-sphere $\mathcal{S}$ in $S^{3}$ bounding closed balls $B_{1}$ and $B_{2}$ such that $\mathcal{S} \cap F$ is a disc, $F \cap B_{1}=F_{1}$, and $F \cap B_{2}=F_{2}$.

Proposition 2.1. [G4] The Murasugi sum of two essential surfaces is essential.

Given a regular projection $\pi$ of a link $l$ we can easily generate spanning surfaces for $l$ which are Murasugi sums of checkerboards (usually not essential). We proceed as follows.

Let $\gamma$ be a simple closed curve in the projection sphere meeting $k$ transversely away from the crossing points and having the property that each arc of $\gamma \backslash k$ joins distinct components of $k \backslash\{$ crossing points $\}$. Let $\Delta$ denote a disc bounded by $\gamma$ and lying below the projection sphere. Then by making the opposite checkerboard colouring choices on each side of $\gamma$, we describe a surface $F$ which is the Murasugi sum along $\Delta$ of two checkerboards. (See Figure 4.)

Definition 2.1. Let $T$ be a checkerboard surface for a link projection $\pi$. Let $\gamma$ and $\Delta$ be as described above, and let $\mathcal{D}$ be the disc in the projection sphere lying above $\Delta$. If $T^{\prime}$ is obtained by making the colouring choices described above so as to agree with $T$ outside $\mathcal{D}$, then we say that $T^{\prime}$ is obtained from $T$ by local dualization on $\mathcal{D}$.

Notice that if $T$ is a checkerboard surface for an alternating link projection, and $T^{\prime}$ is obtained from $T$ by local dualization, then $T^{\prime}$ is the Murasugi sum of two checkerboards with alternating boundary, one with all twists positive and 

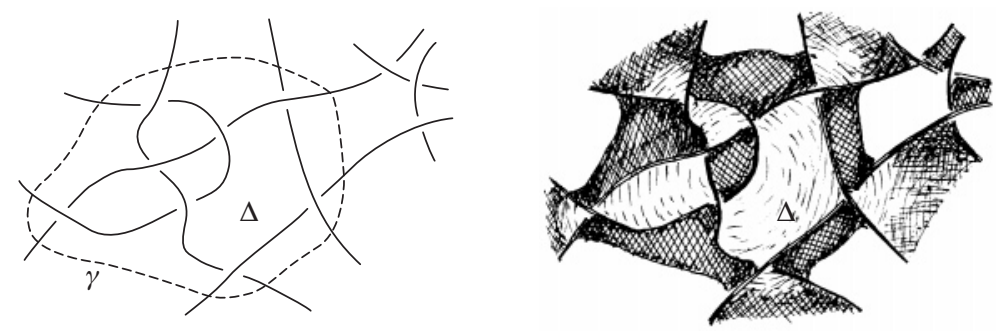

Figure 4.

Murasugi sum of checkerboard surfaces.

the other with all twists negative. This is our basic purpose in using the local dualization operation.

When $\pi$ is a reduced alternating projection we can say precisely when this local dualization produces an essential surface. This result is most easily stated using the language of rigid vertex graphs.

\subsection{Graph descriptions of surfaces}

If $F$ is a checkerboard surface, then $F$ consists of a collection of discs connected by half-twisted bands corresponding to the crossings. Hence, there is a planar graph $\Gamma^{F}$ naturally associated to $F$ : vertices correspond to the discs and edges correspond to the half-twisted bands. Note that if $S$ and $T$ are the two checkerboard surfaces for a link projection $\pi$, then $\Gamma^{S}$ and $\Gamma^{T}$ are dual graphs.

More generally, a given disc and band decomposition of a surface corresponds in a natural way to a rigid vertex graph, by which we mean a graph in which each vertex is regarded as a tiny disc, so that the cyclic order in which the edges are attached is fixed. Each disc in the surface decomposition corresponds to a vertex in this graph, and every twisted band to an edge. We will often wish to label each edge of this graph with a plus or minus sign in order to indicate whether the corresponding half-twist is positive (right-handed) or negative (left-handed). Conversely, any graph in $S^{3}$ with edges labeled in this manner clearly corresponds to a unique surface embedded in $S^{3}$ with a particular disc and band decomposition; for an unlabeled graph, we will follow the convention that all twists are positive. Note that the graph corresponding to a surface is planar if and only if the surface is a checkerboard surface for a diagram of the link that is its boundary.

In keeping with the correspondence between graphs and surfaces, we say that a labeled rigid vertex graph is essential if its corresponding surface is essential.

Corresponding to the notions of Murasugi sum and local dualization for surfaces, there are natural notions of Murasugi sum and local dualization for graphs. Precisely, a graph is a Murasugi sum if there is a 2-sphere whose intersection with the graph is a vertex (viewed as a disc) and which separates it into two nonempty subgraphs, called its Murasugi summands. Clearly the surface corresponding this 
graph is the Murasugi sum of the two surfaces corresponding to these subgraphs.

Given a planar graph $\Gamma$, denote its dual graph by $\Gamma^{*}$. Let $\mathcal{P}$ be the sphere containing $\Gamma$ and $\Gamma^{*}$. Let $\gamma$ be a simple closed curve in $\mathcal{P}$ passing alternately through vertices (here viewed as points) of $\Gamma$ and $\Gamma^{*}$, and let $\mathcal{D}$ be a disk in $\mathcal{P}$ bounded by $\gamma$. Let $\Gamma_{D}^{*}$ and $\Gamma_{D}$ be the planar graphs obtained from $\Gamma^{*} \cap \mathcal{D}$ and $\Gamma \cap\left(\mathbf{R}^{2} \backslash \stackrel{\circ}{\mathcal{D}}\right)$, respectively, by identifying $\gamma$ to a vertex, $\Delta$, which we will view as lying below $\mathcal{P}$.

Definition 2.2. The Murasugi sum (along $\Delta$ ) of $\Gamma_{D}^{*}$ and $\Gamma_{D}$ is said to be obtained from $\Gamma$ by local dualization on $\mathcal{D}$. (See Figure 5a.)

If $F$ is a checkerboard surface with corresponding graph $\Gamma^{F}$ (viewed as lying in the projection sphere), it is clear that the graph obtained from $\Gamma^{F}$ by local dualization on a disc $\mathcal{D}$ corresponds to the surface obtained from $F$ by local dualization on $\mathcal{D}$. (See Figure 5 b.)

Now suppose that $\pi$ is a reduced alternating projection with checkerboard surfaces $S$ and $T$. Suppose $T^{\prime}$ is obtained from $T$ by local dualization on a disc $\mathcal{D}$. Let $S_{\mathcal{D}}$ and $T_{\mathcal{D}}$ be the surfaces associated to $\Gamma_{\mathcal{D}}^{S}\left(=\Gamma_{\mathcal{D}}^{T^{*}}\right)$ and $\Gamma_{\mathcal{D}}^{T}$, respectively. (Hence, $T^{\prime}$ is the Murasugi sum of $S_{\mathcal{D}}$ and $T_{\mathcal{D}}$.) Then, by Proposition $2.1, T^{\prime}$ is essential if $T_{\mathcal{D}}$ and $S_{\mathcal{D}}$ are essential. This observation leads to a simple pair of conditions on $\Gamma^{T}$ and $\mathcal{D}$ which will ensure that $T^{\prime}$ is essential.

Lemma 2.3. $T^{\prime}$ is essential if both of the following conditions hold:

1. At least two edges of $\Gamma^{T} \cap \mathcal{D}$ emanate from each vertex lying on $\gamma(=\partial \mathcal{D})$. (Equivalently, no edge of $\Gamma^{S} \cap \mathcal{D}$ has both endpoints lying on $\gamma$.)

2. No edge of $\Gamma^{T} \cap\left(\mathbf{R}^{2} \backslash \stackrel{\circ}{\mathcal{D}}\right)$ has both endpoints lying on $\gamma$. (Equivalently, at least two edges of $\Gamma^{S} \cap\left(\mathbf{R}^{2} \backslash \stackrel{\circ}{\mathcal{D}}\right)$ emanate from each vertex lying on $\gamma$.)

Proof. As noted, it suffices to check that $S_{\mathcal{D}}$ and $T_{\mathcal{D}}$ are essential. It is easy to check that this is equivalent to there being no loops (edges with both endpoints at a common vertex) in the corresponding graphs $\Gamma_{\mathcal{D}}^{S}$ and $\Gamma_{\mathcal{D}}^{T}$. (A nugatory crossing corresponds to either a loop or an isthmus. Isthmuses may be removed by an isotopy of the surface to yield a checkerboard surface for an alternating projection, which will then be reduced if there are no loops.) Conditions (1) and (2) precisely exclude loops. 

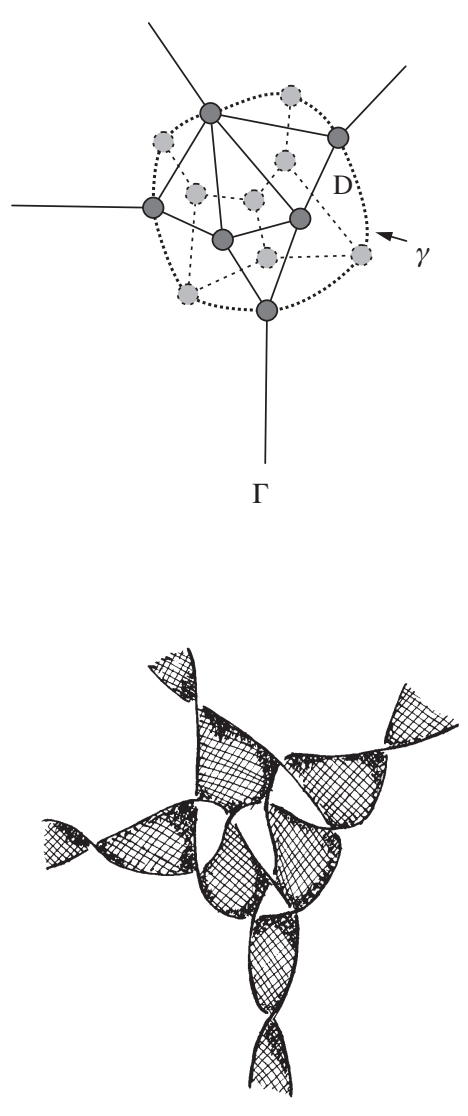

F

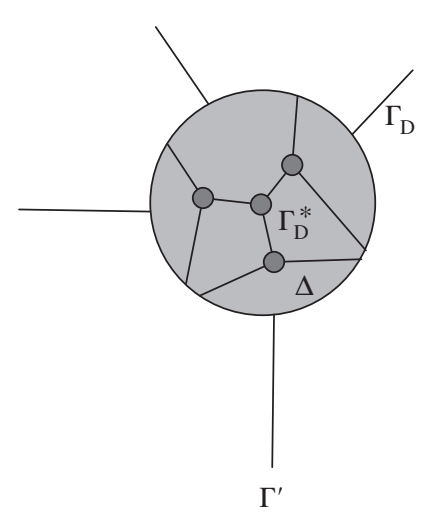

a

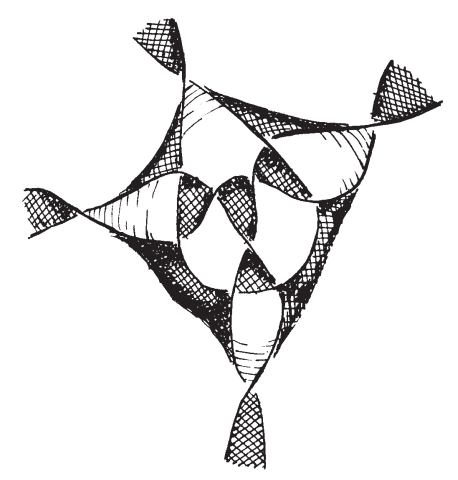

$\mathrm{F}^{\prime}$

b

Figure 5.

Local dualization of graph and corresponding surface.

\section{Laminations}

The basic concepts needed to understand laminations and branched surfaces are laid out in the introductory sections of [GO]. We include the following definition for ease of reference.

Definition 3.1. A lamination is essential if it is fully carried by a branched surface $\Sigma$, with fibred neighborhood $N(\Sigma)$, satisfying the following properties :

1. $\Sigma$ contains no Reeb branched surfaces.

2. $M \backslash \stackrel{\circ}{N}(\Sigma)$ is irreducible, and $\partial M \backslash \stackrel{\circ}{N}(\Sigma)$ is incompressible in $M \backslash \stackrel{\circ}{N}(\Sigma)$. 


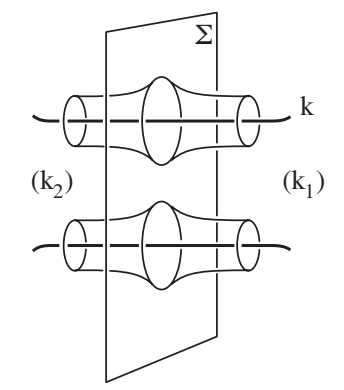

Figure 6 .

Persistent branched surface in composite knot complement.

3. $\partial_{h} N(\Sigma)$ is incompressible and $\partial$-incompressible in $M \backslash \stackrel{\circ}{N}(\Sigma)$, there are no monogons in $M \backslash \stackrel{\circ}{N}(\Sigma)$, and no component of $\partial_{h} N(\Sigma)$ is a sphere or disc properly embedded in $M$ (equivalently, $\Sigma$ carries no sphere or disc).

4. $\Sigma$ has no discs or half-discs of contact.

Remark. A single branched surface may carry many different laminations. We construct essential branched surfaces which carry infinite families of laminations, each lamination intersecting $\partial N(k)$ in curves of a different slope. We do this by adding a disc to the spanning surface as described in Section 3.2

\subsection{Composite knots satisfy strong property $\mathbf{P}$}

For a proof that every composite knot satisfies Property P, see Rolfsen [Ro], p. 281. To prove Strong Property P, we use the decomposition of a composite knot into non-trivial factors to construct a persistent lamination in its complement.

Theorem 3.1. If $k$ is a composite knot, then $S^{3} \backslash k$ contains a persistent lamination.

Proof. Let $\mathcal{S}$ be a sphere separating $k$ into two factors, $k_{1}$ and $k_{2}$. (That is, $S$ intersects $k$ in two points, and $\left(S^{3}, k\right)=\left(S^{3}, k_{1}\right) \sharp_{\mathcal{S}}\left(S^{3}, k_{2}\right)$.) Let $F_{1}$ be the (smooth) surface constructed by attaching to $\mathcal{S} \backslash \stackrel{\circ}{N}(k)$ a tube which follows $k_{1}$. Note that $F_{1}$ would simply be the boundary of a tubular neighborhood of $k_{1}$ if $k_{2}$ were collapsed to a point. (For this reason, $F_{1}$ is often called a "swallow-follow" surface.) Similarly, let $F_{2}$ be the surface constructed by attaching to $\mathcal{S} \backslash \stackrel{\circ}{N}(k)$ a tube which follows $k_{2}$. Let $\Sigma$ be the branched surface $F_{1} \cup F_{2}$ (see Figure 6), and let $\lambda$ be the lamination obtained by splitting $\Sigma$ along $F_{1} \cap F_{2}$. (Thus, $\lambda$ consists of a pair of closed surfaces and is fully carried by $\Sigma$.) We claim that $\lambda$ is persistent.

Let $M_{r}$ be the manifold obtained by Dehn surgery on $k$ with slope $r$. To prove 
the claim, we must show that $\lambda$ is essential in $M_{r}$ for every rational number $r$; hence, we must show that $\Sigma$, viewed as a subset of $M_{r}$, satisfies conditions $1-4$ in Definition 3.1.

Conditions 1 and 4 are clear by construction. To verify 2 and 3, observe that $M_{r} \backslash \stackrel{\circ}{N}(\Sigma)$ consists of three components. The component containing the Dehn filling is a solid torus. Since its vertical boundary consists of two annuli which are meridional to $k$ in $S^{3}$, a meridional disc in $M_{r}$ for this solid torus intersects the core of this vertical boundary at least twice. Hence, this disc cannot be a monogon or a compression of $\partial_{h} N(\Sigma)$. Each of the other components of $M_{r} \backslash \stackrel{\circ}{N}(\Sigma)$ is homeomorphic to one of $S^{3} \backslash \stackrel{\circ}{N}\left(k_{i}\right), i=1,2$. Since $k_{1}$ and $k_{2}$ are both non-trivial knots, we see that conditions 2 and 3 are satisfied for these regions as well.

Corollary 3.1. If $k$ is a composite knot, then $k$ satisfies Strong Property P.

Remark. The fundamental idea of this construction, which is to create two meridional cusps in the branched surface, evolved from an idea of Menasco, who showed that the existence of a pair of non-isotopic meridions ensures that a closed incompressible surface in a knot complement remains essential in the manifold produced by every non-trivial surgery on this knot; see [M1], Theorem 4. (Also cf.: [M2]; [M3]; [GO], Theorem 5.3; [GK]; [cD95], Proposition 3.1; [D2], Corollary to Theorem 1; and $[\mathrm{B}]$.)

\subsection{Persistent laminations from disc decompositions}

With acknowledgement to Definition 1.6 of [G6], we make the following definition.

Definition 3.2. Let $k$ be a knot in $S^{3}$, let $M=S^{3} \backslash \stackrel{\circ}{N}(k)$, and let $F$ be a spanning surface for $k$. Suppose $\mathcal{D}$ is an oriented disc in $M$, with its interior in $\stackrel{\circ}{M} \backslash F$ and its boundary in $\partial M \cup F$, such that $\partial D \cap(\partial M \backslash F)$ is minimal (with respect to isotopy of $\mathcal{D}$ ) and consists of an even number of arcs. (In other words, $\partial D$ "crosses $k$ " an even number of times.) Choose a normal direction for $F$ along $\partial \mathcal{D}$. Then, as described in [G6], a branched surface $\Sigma$ may be constructed from the union of $F$ and $\mathcal{D}$ by choosing the branching direction at $\partial \mathcal{D}$ so that the normal directions to $\mathcal{D}$ and $F$ are consistent. (See Figure 7a.) $\Sigma$ is said to be obtained from $F$ by a disc decomposition on $\mathcal{D}$.

Remark. Our definition is a special case of the definition of Gabai [G6] except that we do not require that $F$ be globally orientable.

Remark. The two choices of orientation for $F$ along $\partial D$, relative to that of $\mathcal{D}$, generally give different results. 


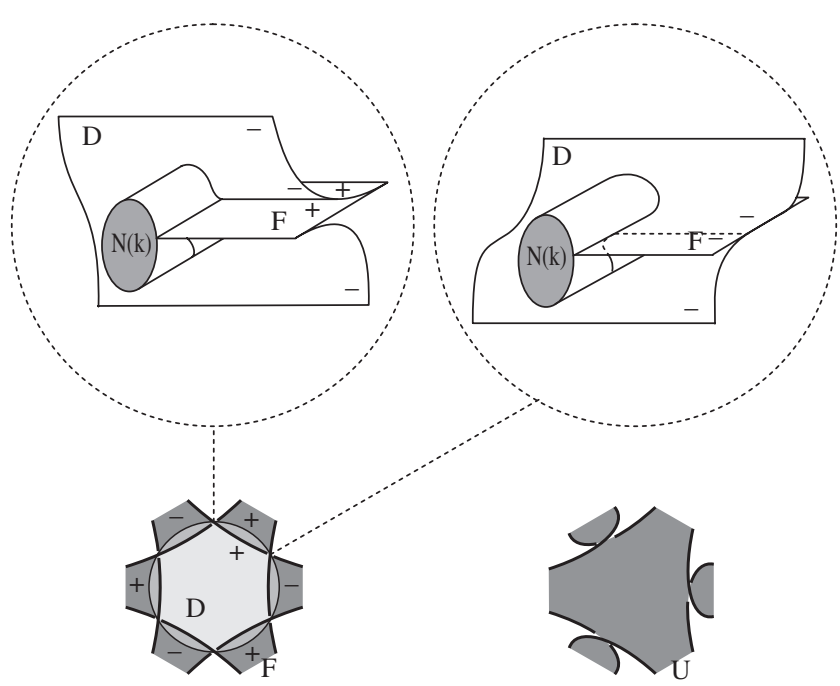

a

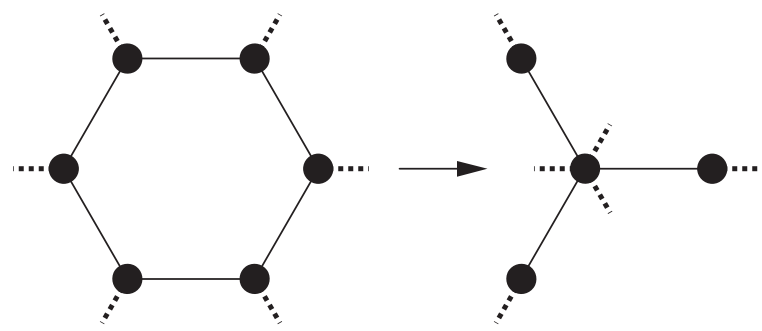

b

Figure 7.

Disc decomposition.

We will further restrict attention to disc decompositions of the following type.

Definition 3.3. Let $F$ be a checkerboard surface for some reduced alternating projection (or else the Murasugi sum of two such checkerboards) and suppose that $\mathcal{D}$ lies on one side of the projection plane and $\partial \mathcal{D}$ corresponds to an innermost simple cycle of even length in $\Gamma^{F}$ (or one of its two Murasugi summands). Call the corresponding disc decomposition simple. Call a simple disc decomposition positive or negative according to whether the disc intersects positive (right-handed) or negative (left-handed) crossings.

As first noted in [G6], a branched surface produced by a simple disc decompo- 
sition continues to "look like" a surface. (See Figure 7a.)

We formalize this observation with the following definition.

Definition 3.4. Let $k$ be a knot, let $M=S^{3} \backslash \stackrel{\circ}{N}(k)$, and let $\Sigma$ be a branched surface in $M$. Suppose that $(\partial M \backslash \stackrel{\circ}{N}(\Sigma)) \cup \partial_{v} N(\Sigma)$ is the union of disjoint annuli; refer to this set as $A(\Sigma)$. Let $U$ be a surface in $S^{3}$ with boundary $l$ (not necessarily connected) and fibred neighborhood $N(U) \simeq U \times I$. Let $M^{\prime}=S^{3} \backslash \stackrel{\circ}{N}(l)$ and $A(U)=\partial M^{\prime} \backslash \stackrel{\circ}{N}(U)$. We say that $\Sigma$ is of spanning type with underlying surface $U$ if there is a homeomorphism

$$
\left(M \backslash \stackrel{\circ}{N}(\Sigma), \partial_{h} N(\Sigma), A(\Sigma)\right) \longrightarrow\left(M^{\prime} \backslash \stackrel{\circ}{N}(U), \partial_{h} N(U), A(U)\right) .
$$

Remark. Heuristically, a branched surface of spanning type is simply a branched surface which looks like a spanning surface for a link if the observer squints and ignores the difference between arcs of "cusp points" coming from the branching locus and arcs of the knot $k$. A spanning surface for $k$ is clearly a special case of a branched surface of spanning type; it is its own underlying surface.

Suppose that a branched surface $\Sigma$ is obtained from a surface $F$ by a simple disc decomposition. Let $\gamma$ be the cycle in $\Gamma^{F}$ corresponding to $\partial D$. Let us say that two vertices of $\gamma$ have the same parity if they are an even distance apart along $\gamma$, and let us divide the alternate vertices of $\gamma$ accordingly into two equivalence classes. Then, as shown in Figure 7b, the underlying surface of $\Sigma$ corresponds to the graph obtained from $\Gamma^{F}$ by identifying one equivalence class of vertices of $\gamma$ to a single vertex and then collapsing each resulting pair of parallel edges, with the choice of vertices to identify determined by the relative orientation of $F$ along $\partial \mathcal{D}$.

If $F$ is a Murasugi sum of checkerboard surfaces with reduced alternating boundary, and no two identified vertices are joined by a single edge, then the underlying surface of $\Sigma$ will also be a Murasugi sum of checkerboard surfaces with reduced alternating boundary, and therefore essential. This motivates the following definition.

Definition 3.5. A good cycle in a uniformly labeled planar summand of a graph is an innermost cycle of even length with the property that no two vertices of the same parity are connected by an edge. We will call a simple disc decomposition good if the cycle corresponding to $\partial \mathcal{D}$ is good.

Lemma 3.1. Suppose the branched surface $\Sigma$ is obtained by a good disc decomposition from a surface $F$ which is a Murasugi sum of checkerboard surfaces with reduced alternating boundary $k$. Then $\Sigma$ is essential. 
Proof. The proofs of Lemma 1.6 and Proposition 1.9 of [R] apply to this situation (with suitable modification of the boundary slopes obtained), showing that $\Sigma$ fully carries a lamination, contains no Reeb components, and has no discs or half-discs of contact. Let $M=S^{3} \backslash \stackrel{\circ}{N}(k)$. Since $\Sigma$ is of spanning type, it is clear that $M \backslash \stackrel{\circ}{N}(\Sigma)$ is irreducible. Furthermore, as noted above, the conditions on a good disc decomposition imply that the underlying surface of $\Sigma$ is essential. It follows that $\partial M \backslash \stackrel{\circ}{N}(\Sigma)$ is incompressible in $M \backslash \stackrel{\circ}{N}(\Sigma), \partial_{h} N(\Sigma)$ is incompressible and $\partial$ incompressible in $M \backslash \stackrel{\circ}{N}(\Sigma)$, there are no monogons in $M \backslash \stackrel{\circ}{N}(\Sigma)$, and no component of $\partial_{h} N(\Sigma)$ is a sphere or disc.

Moreover, $\Sigma$ satisfies the conditions of Theorem 2.3 of $[\mathrm{R}]$, from which we deduce the existence of a family of persistent laminations.

Theorem 3.2. Suppose the branched surface $\Sigma$ is obtained by a good disc decomposition from a surface $F$ that is a Murasugi sum of checkerboard surfaces with reduced alternating boundary $k$. Let $r$ denote the slope of the simple closed curve $\Sigma \cap \partial N(k)$. Then $\Sigma$ fully carries a set $\mathfrak{L}$ of persistent laminations such that for any rational number $s$ in

$$
I= \begin{cases}{[r, \infty)} & \text { if the disc decompostion is positive } \\ (-\infty, r] & \text { if the decomposition is negative }\end{cases}
$$

there is a lamination in $\mathfrak{L}$ which meets the boundary torus of $M$ transversely in parallel curves of slope $s$.

Proof. Lemma 3.1 guarantees that $\Sigma$ is essential and Proposition 1.9 of [R] guarantees that there are no planar surfaces of contact. The remaining hypotheses of Theorem 2.3 of $[R]$ are satisfied by construction. Since the boundary of the decomposing disc for a positive (respectively, negative) good disc decomposition intersects only positive (respectively, negative) crossings, the coefficients of $\frac{x}{x+1}$ and $x$ in the slope formula of Theorem $2.3[\mathrm{R}]$ are \pm 1 (respectively, $\mp 1$ ), from which the result follows.

Corollary 3.2. Suppose the knot $k$ possesses a spanning surface which is a Murasugi sum of checkerboard surfaces with reduced alternating boundary and which contains good cycles of both positive and negative type. Then each manifold obtained by finite Dehn surgery on $k$ contains an essential lamination.

In section 4 we will show that most nontorus checkerboard alternating knots possess such spanning surfaces.

Remark. We note that the above definitions and theorems are certainly not stated in full generality. Disc decompositions which are not simple, decompositions 


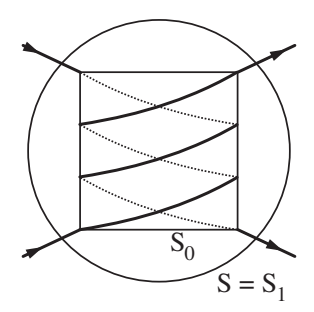

Figure 8 .

$R\left(\frac{1}{3}\right)$

by surfaces other than discs, and decompositions of branched surfaces or less specialized spanning surfaces may be considered and, in some cases, produce similar results for knots we have not discussed.

\subsection{Persistent laminations from rational tangles}

Delman [D2] shows how a persistent lamination may be constructed using a rational tangle decomposition of a knot. We review the necessary elements of this construction. Recall that the lamination constructed by this method is disjoint from a tubular neighborhood of the knot, and persistence means that it remains essential in the manifold obtained by every finite Dehn filling.

A tangle $(B, t)$ is a pair of disjoint arcs $t$ properly embedded in a ball $B$. We say that a knot contains the tangle $(B, t)$ if there is a sphere $\mathcal{S}$ which intersects the knot transversely in four points and bounds this tangle on one side. Consider a collar of $\mathcal{S}$ in $B$ (that is, a neighborhood homeomorphic to $\mathcal{S} \times I$ ), and let $\mathcal{S}_{x}$ denote the level corresponding to $\mathcal{S} \times\{x\}$, with $\mathcal{S}=\mathcal{S}_{1} .(B, t)$ is a rational tangle if $t$ can be isotoped to lie in this collar, intersecting each level $\mathcal{S}_{x}, 0<x \leq 1$, transversely in four points and the level $\mathcal{S}_{0}$ in a pair of arcs. This pair of arcs (and hence the rational tangle) may be described by a rational number, or slope, which is obtained either by viewing $\mathcal{S}_{0}$ as a square "pillowcase" with the endpoints of the arcs at the corners or, more formally, lifting the 2-sphere to its branched covering by $\mathbb{R}^{2}$, branched at these endpoints. (See Figure 8 .) The rational tangle of slope $r$ is denoted by $R(r)$.

Note that in order to assign a well-defined slope, a convention on which arcs have slope $\infty$ must be established.

For our purposes, we need only review the construction of an essential lamination in a knot containing the tangle $R\left(\frac{1}{n}\right)$, where $n$ is an odd integer greater than two. Let $k$ be such a knot, and assume, in addition, that $k$ is oriented as in Figure 8. Let $F$ be a spanning surface for $k$ which intersects the separating sphere, $\mathcal{S}$, in arcs of slope $\infty$. We now describe a branched surface without boundary, $\Sigma \subset S^{3} \backslash k$, constructed from these ingredients. It is easy to see that $\Sigma$ fully carries a lamination (refer to [D2] for details). 


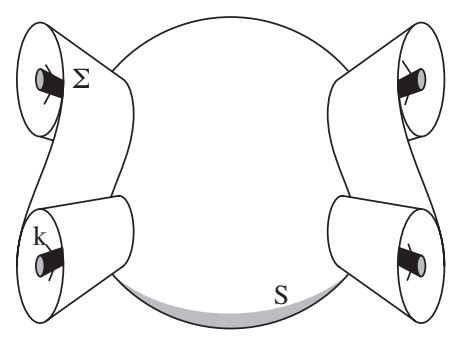

$\mathbf{a}$
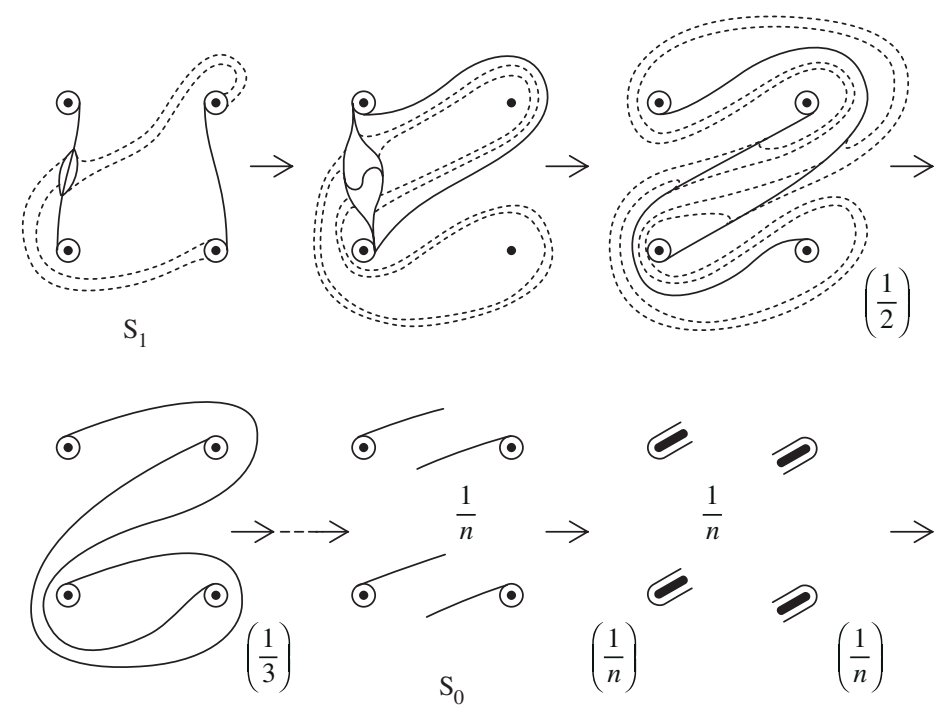

$\mathbf{b}$

Figure 9 .

Branched surface in complement of knot with rational tangle.

In $S^{3} \backslash B$ : $\Sigma$ consists of $\partial N(k)$ together with $F \backslash B$, made tangential to $\partial N(k)$ along its boundary according to an orientation of $k$ and the right-hand rule. (See Figure 9a.)

In $B: \Sigma$ is constructed by specifying its intersections with the "level" spheres $\mathcal{S}_{i}$, proceeding from level $i=1$ down to $i=0$, as shown in Figure 9b. Note that $\Sigma$ has several saddle singularities; $\Sigma$ is "finished off" with two minima in a level just below $\mathcal{S}_{0}$ (which is why $n$ must be odd).

¿From a point of observation outside $B, \Sigma$ looks like a spanning surface for a simpler link, namely the surface $E$ obtained by taking the union of $F \backslash B$ with an 

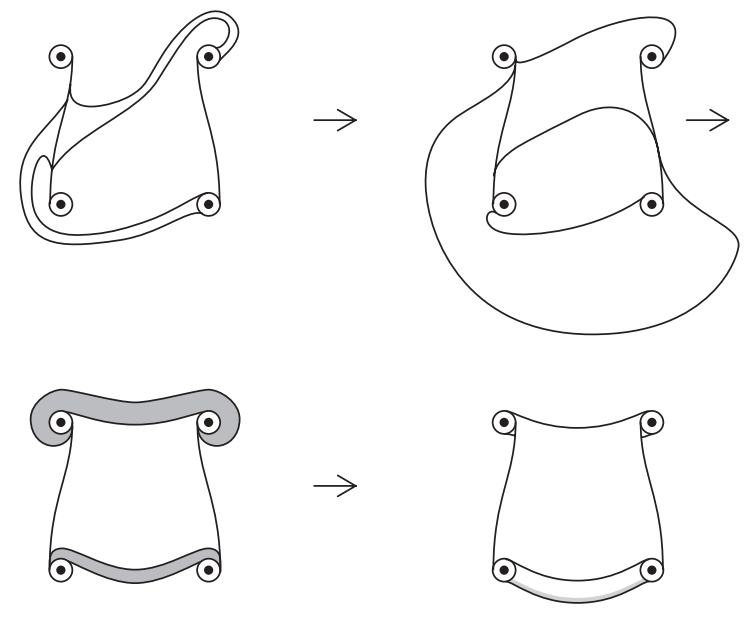

View looking into B from outside

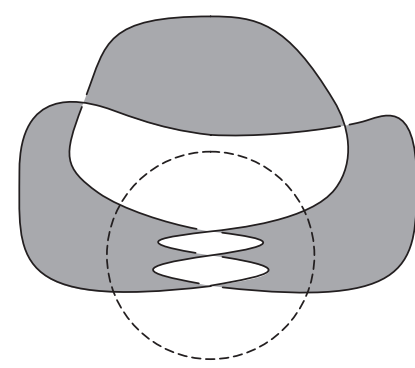

The spanning surface $F$

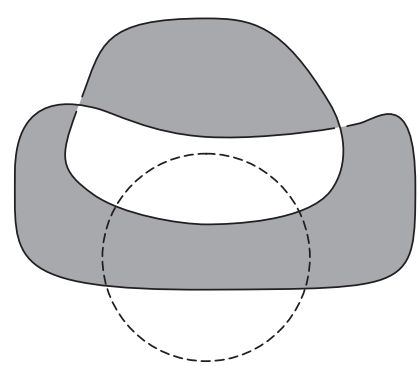

The enveloping surface $\mathrm{F}$

Figure 10.

Visualizing the enveloping surface.

untwisted band in $B$ (that is, a band whose boundary is a tangle of slope 0 ). (See Figure 10.)

Precisely, let $C$ be the component of $S^{3} \backslash \stackrel{\circ}{N}(\Sigma)$ which does not contain $k$ and has non-empty intersection with $\mathcal{S}$. Denote $\partial E$ by $l$, and let $N(l)$ be a tubular neighborhood of $l$. Let $M^{\prime}=S^{3} \backslash \stackrel{\circ}{N}(l)$ and $A(E)=\partial N(l) \backslash \stackrel{\circ}{N}(E)$. Then $C$ looks like the complement of $F^{\prime}$ in $M^{\prime}$ in the sense that there is a homeomorphism

$$
\left(C, \partial_{h} N(\Sigma), \partial_{v} N(\Sigma)\right) \rightarrow\left(M^{\prime}, \partial_{h} N(E), A(E)\right) .
$$

$E$ is called the enveloping surface for $\Sigma$. The enveloping surface plays a role in the current construction analagous to that played by the underlying surface for 
a branched surface of spanning type. In particular, it is proven in [D2] that $\Sigma$ is essential and fully carries a persistent lamination if and only if its enveloping surface is essential.

For alternating knots, we thus obtain the following:

Theorem 3.3. Let $k$ be a checkerboard knot with reduced alternating projection $\pi$, and let $T$ be the unorientable checkerboard surface for $\pi$. Suppose there is a disk $\mathcal{D}$ in the projection sphere whose intersection with $\pi$ is the projection of a rational tangle $R\left(\frac{1}{n}\right), n$ odd and $\geq 3$, where the intersection of $T$ with the separating sphere is taken to have slope $\infty$. Furthermore, suppose that $\mathcal{D}$ and $T$ satisfy condition 2 of Lemma 2.3. Then the complement of $k$ contains a persistent lamination (without boundary).

Proof. Construct the branched surface $\Sigma$ from $R\left(\frac{1}{n}\right)$ and $T$ as described above. (Note that the orientation of $k$ is correct, since $T$ is dual to the Seifert surface.) The enveloping surface for $\Sigma$ is simply the surface $T_{\mathcal{D}}$ corresponding to the graph obtained from $\Gamma^{T}$ by identifying $\mathcal{D}$ to a vertex. By Lemma 2.3, $T_{\mathcal{D}}$ is essential. Hence, by the Corollary to Theorem 1 of [D2], $\Sigma$ is essential and fully carries a persistent lamination.

Corollary 3.3. Suppose $k$ is a knot satisfying the hypotheses of Theorem 3.3. Then every manifold obtained by finite Dehn surgery on $k$ contains an essential lamination.

In section 4 we show that any prime nontorus alternating knot which does not have a spanning surface satisfying the hypothesis of Corollary 3.2 does have a spanning surface and tangle satisfying the hypothesis of Corollary 3.3 .

\section{Proof of the main theorem}

Let $k$ be a prime nontorus checkerboard alternating knot. Let $S$ denote the orientable checkerboard surface and $T$ the nonorientable one for a reduced alternating projection of $k$. We begin with a preliminary lemma.

Lemma 4.1. For $k, S$, and $T$ as above, there exist simple cycles $\alpha$ and $\beta$ in $\Gamma^{T}$ with the following properties.

1. $\alpha$ is innermost.

2. $\alpha \backslash \beta$ is connected and has a nonzero even number of edges.

3. Let $\mathcal{D}_{\beta}$ be the disc bounded by $\beta$ which does not contain $\alpha$. Then no two vertices of $\overline{\alpha \backslash \beta}$ with the same parity are joined by an edge not contained in $\alpha \cup \mathcal{D}_{\beta}$.

4. No two vertices of $\beta$ are joined by a single edge not contained in $\mathcal{D}_{\beta}$. 
Proof. Since $T$ is nonorientable, $\Gamma^{T}$ contains a cycle (and hence an innermost one) of odd length. Therefore there is a vertex $v$ in $\Gamma^{S}$ of odd valence. Since $v$ has odd valence it must connect to one of its neighbours, $w$ say, by an odd number of edges.

Let $\alpha$ and $\beta$ be the cycles dual to $v$ and $w$ respectively. Since $k$ is not a torus knot, $\alpha$ and $\beta$ are distinct. Furthermore, since $k$ is prime, both $\alpha$ and $\beta$ are simple, and $\beta$ cannot contain all edges of $\alpha$.

If $\beta$ meets $\alpha$ in a disconnected set, then we rechoose $\beta$ as follows. We note that $\alpha \backslash \beta$ can be viewed as a union of disjoint open intervals. Let $x$ and $y$ be the endpoints of one such interval. Suppose $\alpha$ is the simple cycle of edges $a_{1} a_{2} \ldots a_{2 m+1}$, where $a_{1} a_{2} \ldots a_{r}$ denotes the sequence of edges in $\alpha \backslash \beta$ describing a path from $x$ to $y$. Similarly, let $b_{1} b_{2} \ldots b_{s}$ denote the sequence of edges in $\beta \backslash \alpha$ joining $x$ to $y$. If $r$ is odd, then rechoose $\beta$ to be the cycle $a_{1} a_{2} \ldots a_{r} b_{s} \ldots b_{2} b_{1}$. Otherwise, rechoose $\beta$ to be the cycle $a_{r+1} \ldots a_{2 m+1} b_{1} \ldots b_{s}$. In either case, $\alpha \backslash \beta$ is a non-empty path with an even number of edges.

Note that $\alpha$ and $\beta$ satisfy conditions 1 and 2 , but possibly not 3 and 4 .

Let $\mathcal{D}_{\alpha}$ be the disc bounded by $\alpha$ which does not contain $\beta$. (Note that the interior of $\mathcal{D}_{\alpha}$ contains no edges of $\Gamma^{T}$, since $\alpha$ is an innermost cycle.) Suppose condition 3 fails to hold. This means there is an edge in the complement of $\mathcal{D}_{\alpha} \cup \mathcal{D}_{\beta}$ whose endpoints are joined in $\alpha$ by a path $\rho$ of even length. Let $e$ be such an edge which is innermost in the sense that the disc $\mathcal{D}$ bounded by $e$ together with $\rho$ in the complement of $\stackrel{\circ}{\mathcal{D}}_{\alpha}$ contains no other such edge. Then replace $\mathcal{D}_{\beta}$ with the complement of (the interior of) $\mathcal{D} \cup \mathcal{D}_{\alpha}$, and replace $\beta$ with the simple cycle bounding this new $D_{\beta}$. (Hence, after this modification, $\alpha \backslash \beta=\stackrel{\circ}{\rho}$.) Now conditions 1 and 2 are clearly still satisfied. Furthermore, since $e$ was chosen innermost, conditions 3 and 4 also hold.

Finally, suppose that $\alpha$ and $\beta$ are innermost and satisfy conditions 1-3 but not 4. Note that condition 2 guarantees that any edge not in $D_{\beta}$ and yet joining two vertices of $\beta$ is not contained in $\alpha$. Thus, the following procedure does not violate conditions 1-3 and may be repeated until 4 is satisfied: if there is a subpath of $\beta$ which connects vertices joined by a single edge outside $D_{\beta}$, then replace that subpath with this edge.

Theorem 4.1. Every manifold obtained by finite surgery on a nontorus alternating knot contains an essential lamination.

Proof. By 3.1, we may assume that $k$ is prime. Without loss of generality, suppose that the edges of $\Gamma^{T}$ are negatively labelled.

Choose simple cycles $\alpha$ and $\beta$ in $\Gamma^{T}$ satisfying properties 1-4 of Lemma 4.1. Let $\gamma$ be the simple closed curve which is disjoint from the edges of $\Gamma^{S} \cup \Gamma^{T}$ and which passes alternately through vertices of $\beta$ and vertices of $\Gamma^{S}$ lying outside $\mathcal{D}_{\beta}$; let $\mathcal{D}$ be the disc bounded by $\gamma$ that contains $\mathcal{D}_{\beta}$. The local dualization of $\Gamma^{T}$ on $\mathcal{D}$ is the Murasugi sum of the two planar graphs $\Gamma_{\mathcal{D}}^{T}$ and $\Gamma_{\mathcal{D}}^{S}$, where all edges of 

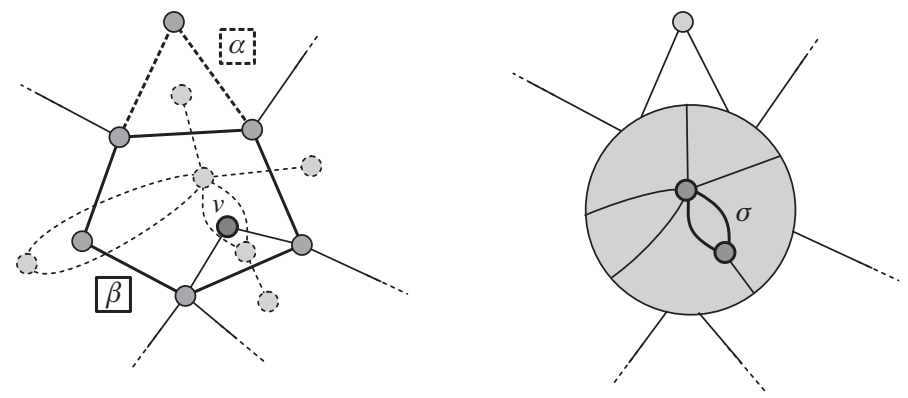

Figure 11.

Good positive cycle in Case 1.

$\Gamma_{\mathcal{D}}^{T}$ are negatively labeled and all edges of $\Gamma_{\mathcal{D}}^{S}$ are positively labeled. Lemma 2.3 and condition (4) of Lemma 4.1 guarantee that both of the surfaces, $T_{\mathcal{D}}$ and $S_{\mathcal{D}}$, corresponding to these graphs, are essential. Let $T^{\prime}$ denote, as before, the essential surface obtained by local dualization of $T$ along $\mathcal{D}$.

Conditions 1 and 2 of Lemma 4.1 guarantee that $\alpha$ collapses in $\Gamma^{T^{\prime}}$ to an innermost cycle, $\alpha^{\prime}$, of even length, and condition 3 of Lemma 4.1 ensures that $\alpha^{\prime}$ is good. Hence, $T^{\prime}$ admits a good negative disc decomposition.

We next look in the summand $\Gamma_{\mathcal{D}}^{S}$ for a good, positively labeled cycle. If we find one (and in most cases we do), then application of Corollary 3.2 completes the proof. If we don't find one, then we demonstrate instead that the hypothesis of Corollary 3.3, from which the theorem also follows, is satisfied. We divide the proof into three cases.

Case 1. There is a vertex, $v$, of $\Gamma^{T}$ which lies in the interior of $\mathcal{D}$.

Let $\sigma$ be the cycle dual to $v$. Since $v$ is in the interior of $\mathcal{D}$, no vertex of $\sigma$ lies on $\gamma$. Hence $\sigma$ is a cycle of $\Gamma_{S}$ and, since $S$ is orientable, must be of even length. Furthermore, no two vertices of $\sigma$ an even distance apart are joined by a single edge not in $\sigma$, since these edges would also produce an odd cycle in $\Gamma_{S}$. Thus $\sigma$ is good. (See Figure 11.)

Case 2. No vertex of $\Gamma^{T}$ lies in the interior of $\mathcal{D}_{\beta}$, and $\beta$ has more than two vertices.

In this case, the subgraph of $\Gamma^{T}$ contained in $\mathcal{D}$ must consist of families of edges parallel to those of $\beta$. Let $x$ denote the vertex of $\Gamma_{\mathcal{D}}^{S}$ dual to the innermost simple cycle traversing the vertices of $\beta$ (which is well-defined since, by assumption, $\beta$ has at least three vertices); let $y$ denote the vertex obtained from $\mathcal{D} . \Gamma_{D}^{S}$ consists of unbranched paths joining $x$ and $y$ (see Figure 12). If any two adjacent paths have odd length, then together they form a positively labeled good cycle.

If not, rechoose $\alpha$ and $\beta$ as follows. Since no two adjacent paths joining $x$ and $y$ have odd parity, there must be at least two paths whose length is even and, consequently, greater than one. Choose $\beta$ to be the cycle of length two formed 

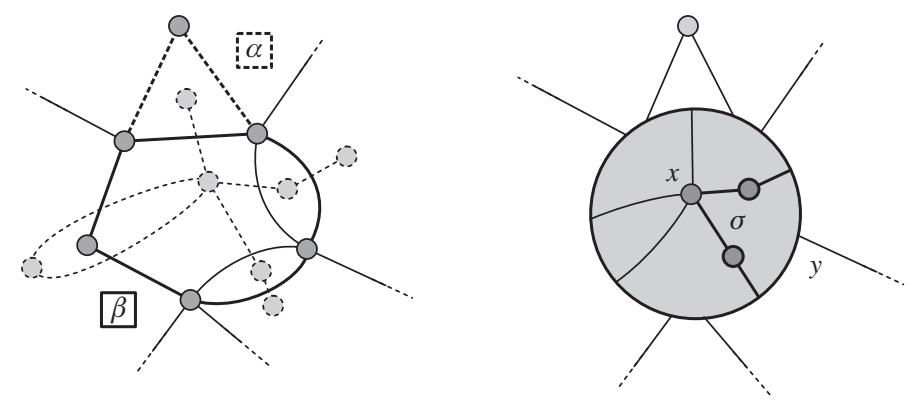

Figure 12.

$\Gamma_{D}^{S}$ in Case 2.
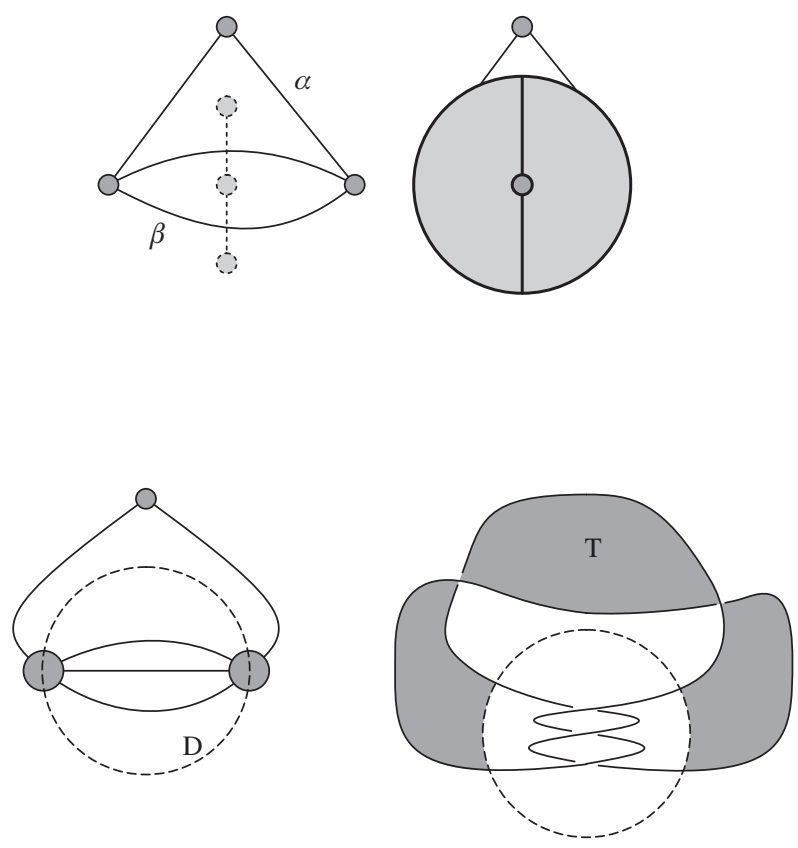

Figure 13.

Rational tangle in Case 3.

by the outermost edges dual to one of these paths; choose $\alpha$ to be the innermost cycle (which will also be of length two) dual to any vertex in the interior (that is, neither $x$ nor $y$ ) of the other. It is easy to see that this choice of $\alpha$ and $\beta$ satisfies the conditions of Lemma 4.1. Furthermore, after replacing $\mathcal{D}$ with the disc determined by this new choice of $\beta$ and performing local dualization on it, we see that the new $\Gamma_{\mathcal{D}}^{S}$ consists entirely of a good cycle. 
Case 3. No vertex of $\Gamma^{T}$ lies in the interior of $\mathcal{D}_{\beta}$, and $\beta$ has exactly two vertices.

In this case, the subgraph of $\Gamma^{T}$ bounded by $\mathcal{D}$ consists of a single family of (at least two) parallel edges; hence $\Gamma_{\mathcal{D}}^{S}$ consists of a single cycle. Let $n$ be its length. If $n$ is even, then this cycle is good. If $n$ is odd, then consider the portion of $k$ whose projection lies inside $\mathcal{D}$. This is a rational tangle of type $\frac{1}{n}$ when viewed so that the surface $T$ cuts its boundary in arcs of slope $\infty$. Since $n$ is odd, Corollary 3.3 applies. (See Figure 13.)

Corollary 4.1. Alternating knots satisfy Strong Property P; in particular, they satisfy Property $P$.

\section{References}

[B] M. Brittenham, Persistently Laminar Tangles, preprint.

[C] R. Crowell, Genus of Alternating Link Types, Ann. of Math. 69 (1959), 258-275.

[D1] Charles Delman, Essential Laminations and Dehn Surgery on 2-bridge Knots, Topology and its Appl. 63 (1995), 201-221.

[D2] Charles Delman, Constructing Essential Laminations Which Survive All Dehn Surgeries, preprint.

[G1] David Gabai, Foliations and the Topology of 3-Manifolds, J. Diff. Geom. 18 (1983), 479-536.

[G2] David Gabai, Foliations and the Topology of 3-Manifolds II, J. Diff. Geom. 26 (1987), 461-478.

[G3] David Gabai, Foliations and the Topology of 3-Manifolds III, J. Diff. Geom. 26 (1987), 479-536.

[G4] David Gabai, The Murasugi Sum is a Natural Geometric Operation I, Cont. Math. 20 (1983), 131-145.

[G5] David Gabai, The Murasugi Sum is a Natural Geometric Operation II, Cont. Math. 44 (1985), 93-100.

[G6] David Gabai, Foliations and Genera of Links, Topology 23 (1984), 381-394.

[G7] David Gabai, Eight Problems in the Geometric Theory of Foliations and Laminations on 3-Manifolds, in: William H. Kazez, ed., Geometric Topology 1993 Georgia International Topology Conference, August 2-13, 1993, AMS/IP, Providence, 1997.

[GK] David Gabai and William Kazez, Pseudo-anosov Maps and Surgery on Fibred 2-bridge Knots, Topology and its Applications 37 (1990), 93-100.

[GO] David Gabai and Ulrich Oertel, Essential Laminations in 3-manifolds, Ann. Math. 130 (1989), 41-73.

[M1] William Menasco, Closed Incompressible Surfaces in Alternating Knot and Link Complements, Topology 23(1) (1984), 37-44.

[M2] William Menasco, Constructing Incompressible Branched Surfaces, preprint.

[M3] William Menasco, Branched Surfaces and Knots in $S^{2} \times S^{1}$, preprint.

[MT] William Menasco and Morwen Thistlethwaite, The Tait Flyping Conjecture, Bull. A.M.S. 25(2) (1991), 403-413.

[Mo] Louise Moser, Elementary Surgery along a Torus Knot, Pac. J. Math. 38(3) (1971), $737-745$.

[Mu1] K. Murasugi, On the Genus of the Alternating Knot I, J. Math. Soc. Japan 10 (1958), 94-105.

[Mu2] K. Murasugi, On the Genus of the Alternating Knot II, J. Math. Soc. of Japan 10 (1958), 
$235-248$.

[R] Rachel Roberts, Constructing Taut Foliations, Comment. Math. Helvetici 70 (1995), 516545 .

[Ro] Dale Rolfsen, Knots and Links, Publish or Perish, Houston, 1990.

Charles Delman

Department of Mathematics

Eastern Illinois University

Charleston, IL 61920

USA

e-mail: cfcid@eiu.edu
Rachel Roberts

Department of Mathematics

Washington University

St Louis, MO 63130

USA

e-mail: roberts@math.wustl.edu

(Received: May 20, 1998) 